\title{
Argumentation-Based Group Decision Support for Collectivist Communities
}

\author{
Marijke Coetzee \\ Academy for Computer Science and Software engineering, \\ University of Johannesburg, South Africa \\ marijkec@uj.ac.za
}

\begin{abstract}
In collectivist communities, decisions are taken by groups of people who prefer to consider the opinions of their in-group. For them it is important to reach group consensus by focusing on the group's preferences and goals. Such decision processes can be supported by multi-criteria decision analysis that identifies sets of objectives, representing subjective values used in decision making, to better generate recommendations. Recently, several attempts have been made to explain and suggest alternatives in decision-making problems by using arguments. Argumentation theory is the process of bringing together arguments so that conclusions can be justified and explained. Each potential decision usually has arguments for or against it, of various strengths. For collectivist communities, the non-monotonicity of argumentation theory is useful as it supports an adaptive decision-making style. The fact that the opinions of group members can be evaluated and replaced, if they are found lacking via a group opinion strategy, fits well with collectivist decision-making. This paper proposes a framework that allows a group of users, belonging to a collectivist and mostly rural community, to share their opinions when making decisions such as buying goods in bulk in order by incorporating their cultural beliefs in the system design.
\end{abstract}

Keywords: Collectivist culture, group decisions, multi-criteria decision-making, argumentation.

\section{$1 \quad$ Introduction}

In the developing economies of Africa, South America, India and Asia, very small enterprises (VSEs) find it challenging to conduct business supported by ecommerce [1]. VSEs are part of the informal economic sector and have an important role to play in the growth of developing economies. It is therefore vital that these, and other types of VSEs, are supported by technology to enable growth and expansion. To answer this need, recent research projects investigate how to support VSE owners by means of m-commerce applications backed by cloud-based technologies [5] [6] to allow them to conduct their business from a mobile phone. For such applications to be successful, VSE owners should be encouraged to collaborate, and together decided to buy products in bulk from large retail suppliers in order to become more profitable. 
An analysis of data collected in the U.S. (highly individualistic) and Ghana (highly collectivist) points to the fact that culture affects decision making [2]. It is thus important for researchers who are designing decision-making tools to be aware of the fact that individualist are inclined to be more rational, and collectivist are inclined to be more dependent when making decisions. Individualistic consumers would rather evaluate the features of a product before they purchase it, in contrast to collectivists in Asia or Africa who consider factors such as status and symbolism [7]. Therefore the role of culture on group decision-making needs to be investigated as currently, no culturally adapted group decision-making tool exists that incorporates collectivist behavior and beliefs.

In order to address this, this research contributes a framework where humans interact with an application on their mobile phone that accumulates the preferences of all members of a collaborative group and addresses cultural factors when decisions are made. The reasoning model is based on an argumentative multi-criteria decision framework [8] to assist members to make decisions. The focus is to determine a collective choice that will satisfy at most of the goals of the group. Arguments provide the pros and cons to support decisions and are not of equal strengths, which makes it possible to compare pairs of arguments. When arguments are compared, it is done using different principles, it is done on the basis of their relevant strengths and whether they are for or against a choice. The process of argumentative decisionmaking is supported by decision-making strategies that meet the needs of collectivists such as a group opinion strategy and a group customisation strategy.

The paper is structured as follows: The next section gives a motivating example that highlights the environment of the collectivist consumer that is considered by this research. The influence of culture on decisions is described by identifying main cultural approaches and the effect of collectivism on decision-making and social position. Group decision methods are discussed and multi-criteria decision analysis is identified as a promising approach for this problem area. To assist a collectivist decision-maker in his decision processes, argumentation theory can be used with multi-criteria decision analysis to provide not only a decision, but also the explanation to the decision. A framework is presented that illustrates how different group decision strategies are implemented with examples. Finally, the paper is concluded.

\section{Motivating Example}

There is an acute need for culturally-adapted technology to assist VSEs to collaborate more. Today, rural collectivist business owners in Africa, Asia and South America interact in a face-to-face manner with each other in a culturally involved manner to ensure that their businesses will survive in the face of adversity. Figure 1 gives nodes representing VSEs, as well as a large retail supplier of goods. Each of these VSEs operate on their own and buy good from the supplier individually, at more expensive prices. The focus of such a collaboration tool would for example be to assist business owners to group together to buy goods in bulk at a cheaper price from this or any other supplier. 


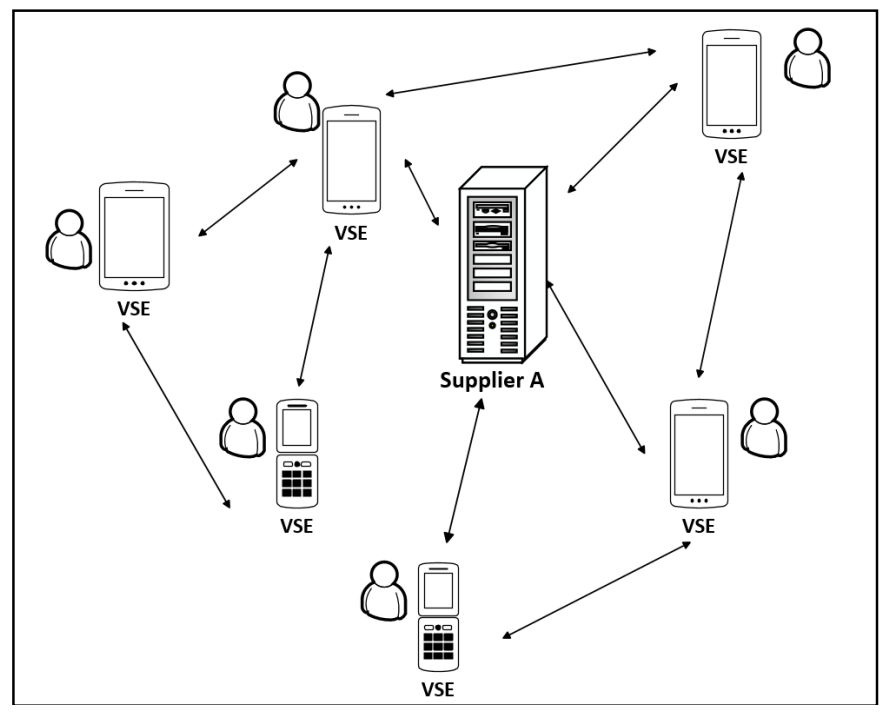

Fig. 1. Very small enterprises collaborating

Due to the size of VSEs and their informal nature, owners tend to run the business and make business decisions based on personal relationships and understandings, where social capital and social ties support and influence all decisions. Their behavior is strongly influenced by their cultural norms and beliefs [9]. This is even more relevant when collaborations occur since it involves committing to and trusting in a relationship with another business owner. In order to be able to implement such systems successfully, the collectivist approach to consumer decision-making needs to be understood for such systems to be useful. The aim is to ensure that participants are comfortable with the manner in which the system enables them to collaborate in a group.

\section{Culture and Decisions}

Culture is generally described as the shared symbols, norms, and values in a social group such as an organisation or country [9]. Hofstede [9] [11] has identified dimensions of national culture of which individualism vs. collectivism is the most prominent. Other dimensions include power distance, uncertainty avoidance, and masculinity vs. femininity. These dimensions are generally applied by other researchers even though Hofstede's model has been criticized as too simplistic and not refined. Yet Hofstede's model has been validated by as many as 140 studies [12], making it useful in many different situations.

Individualist societies are found mainly in North America and much of Western Europe. For them, ties between members of the community are loose where people make a life for themselves and their direct family members only. The interest of a specific person is more important than the interest of the group. Individualists are more goal-oriented and self-motivated, and use guilt and loss of self-respect as 
motivators [9] [11]. Individualists perform their duties if they can gain from it, and see themselves independent from any groups.

Collectivist societies are found mainly in much of the Middle East, Asia, Africa, and South America. People are incorporated into strong, cohesive in-groups from birth. In these groups of people there is concern for the wellbeing of others. A person separated from the group experiences anxiety because of this separation. In-groups protect their members in exchange for loyalty to the in-group. Group interests and goals are more important than individual interests, and individuals conduct themselves in a manner to maintain social harmony. Such individuals acquire the skills and qualities necessary to be a good group member and to keep to tradition. Typical motivators are shame and loss of face [9]. Collectivists enjoy doing what is right for their in-group, and have self-identities that are strongly linked to the features of their group [27].

Current collaborative group decision tools are designed to suit individualistic communities and may thus be less useful to people in many of the collectivist cultures in the world. For example, expert opinion carries more weight in decision-making [17], where collectivists do not necessarily value expert opinion as much as it may be to the detriment of group harmony. Based on a review of collectivists decision-making tendencies that now follows, this research aims to present strategies to be used in the design of decision-making tools for a collectivist audience.

\subsection{Collectivism and Decisions}

Studies have investigated the impact of culture on group decision making [13] [14] [15] and has highlighted that the importance of group opinion, maintenance of consensus, and decisions of people with high social positions in the group cannot be underestimated. Collectivists follow an adaptive and more dependent decision-making style to maintain group harmony where the needs of the group outweigh the needs of individuals' opinion of a group leader.

Group Opinion - Collectivists generally give more weight to the opinions of their in-group than individualists do, and thus these opinions strongly influence their decisions. When collectivists perform an activity in isolation, they feel isolated and are less effective [9]. A group opinion strategy to consider may be to provide the user with the opinions of the other in-group members when they make a decision. [14]. This lessens the user's feelings of isolation, and reduces the time taken to seek advice from members of the in-group regarding their opinions. [14].

Consensus Change - Collectivists values consensus highly. They have less change in consensus between themselves than individualists [13] and rather strive to maintain consensus.

Group Polarization - Polarization is the inclination of individuals in a group setting to hold very different opinions to the group [13]. Polarization is a largely individualistic trait where individuals want to showcase their ideas and influence others to promote self-interest. In contrast, collectivist opinions do not lead to polarization in the group's decision and persuasive arguments. 
Persuasive Arguments - Collectivists experience lower levels of persuasive content from arguments presented, due to the collective and high power distance of the culture [13]. They may rather support the arguments of powerful people in the group than arguments with more merit.

Adaptive Decision-Making - Collectivists adapt their decisions as needed as they are part of a group and are more receptive to social consequences of their decisions. Individualists view themselves as independent and responsible for their decisions [15] and keep to the same decision strategies.

Group Surveillance - Individualists use guilt as a negative motivational force, whereas collectivists use shame of the in-group as a result of their actions [9]. This strategy sets to track the behaviour of an individual or a group of individuals, to either reward or punish them later [14]. Collectivists' identities are strongly tied to that of their in-group and they are want to contribute as team members. An entire group is thus rewarded or punished for the actions of the individual.

Disapproval Conditioning - Individualists make use of positive reinforcement as a motivational strategy, but collectivists are use negative reinforcement to be motivated [14].

Deviation Monitoring - As collectivists desire to fit in with their in-group, they change their behaviours to adapt to the group and situation to ensure they do not stand out [14]. Collectivist cultures agree on what expected behaviour is deemed to be and they are given severe criticism for small deviations from the norm.

Group Customisation - In collectivist cultures it is important to put in-group goals ahead of one's own goals [11]. This means that in-group preferences may out-weigh individual preferences in different circumstances, particularly where members of the same in-group are together. A group customisation strategy can permit one member of an in-group, or perhaps various members, to modify application settings on behalf of the in-group's needs, preferences, and goals [14].

Previous research indicates that the ignorance of such culture differences cause the failure of systems [25]. Currently, many group decision-making tools are designed by individualists in the Western world, who do not consciously set out to focus on the individualistic culture. They are not aware of the features they design in this manner, but when such tools are used by different cultural groups, these same features surface to become problematic.

\subsection{Collectivism and Social Position}

Decisions are not only influenced by culture, but also by the opinions of group leaders. Such leaders are members of a group whose exercises strong influence over the members of the group [16]. In collectivist cultures, members look up to other group members for guidance who have a higher social in the community. Such a leader is trusted by its group members where there is more trust as the social position of the leader increase. Well connected leaders can provide access to a wider range of resources [24] and are beneficial to the group. Such leaders form potential collaborations with others outside the group, leading to potential business opportunities. 
When groups are formed, new members are generally only invited if they are personal friends or well-know to current members [24]. Since current members are held responsible, to a certain degree, for the new member's behaviour, it is crucial that new member should behave according to the norms of the group. Collectivist cultures encourage individuals to relate to those in their in-groups and rely less on others outside the in-group to instill strong loyalty towards the group.

\section{Group Decisions}

A Group Decision can be defined as "a decision problem that is shared by two or more parties who make a choice for which all parties will bear some responsibility" [18]. Group Decision Support System (GDSS) enables the definition of alternatives, the choice of criteria and their weights, performance and final recommendations [17]. In order to obtain a recommendation a complete decision process is supported by formulating and structuring the problem, defining an evaluation model, finding an answer to the problem, and finally constructing a recommendation [19]. Many GDSS tools have been proposed that support brainstorming to generate ideas, blackboards to share information, argumentation models for decision analysis, and multiple criteria decision models for alternative evaluations. Recent research on GDSS implement consensus models that take into account coincidence among preferences, similarity criteria among the solutions obtained from the experts' preferences, and the agreement between the experts' individual opinions and the group opinion using fuzzy measures [26] illustrating the trend in these systems to focus only on rational decision-making. Social parameters such as trust or culture are generally ignored by the research community, leaving a gap to be explored.

Of the available set of GDSS decision models, multi-criteria decision analysis shows promise to support collectivist decision-making. It provides a set of techniques and principles to compare and evaluate alternatives according to criteria [18] [19]. A recommendation is constructed, based on formal preference models defined by users in the decision process, making it possible to explain why a preference is preferred over another. This aspect is relevant to decision-making in collectivist communities, as group members can be given access to the opinions of other group members, to make them more comfortable and effective in their decision-making. A group member can be given a recommendation, and also the reasons that underlie this recommendation by making use of argumentation. Amgoud et al. [8] proposes an approach that explicitly links argumentation to multi-criteria decision making, discussed next.

\section{Argumentation}

When considering classical logical reasoning such as propositional or predicate logic it is inferred that a conclusion can be found to be true despite any new additions made to the set of proposition which supported the conclusion. This is defined as monotonicity, where additional information does not cause conclusions to be modified or withdrawn [19]. For human reasoning this is not natural as people can change 
their minds when new information is made available. Decisions are based on the available information at a specific moment in time, and can be modified at any time. Non-monotonic reasoning formalisms have emerged over time of which argumentation theory is considered by this research. Argumentation theory enables reasoning, by constructing and comparing arguments for and against conclusions [20].

Non-monotonicity arises as new facts enable the construction of new arguments to support new conclusions, or support stronger counter-arguments against existing conclusions. Those arguments are intended to support, explain, or attack statements that can be decision, opinions, preferences, and values. Argumentation thus fits more naturally with human decision-making as people use arguments to support the claims that contribute to these decisions [21] [28]. Each potential decision usually has a set of arguments for or against it, of various strengths. The adoption of argumentation to a decision support system has direct benefits as the user can be provided with a choice that is not only well-founded, but is also accompanied by the reasons underlying the decision. Furthermore, as argumentation-based decision making is similar to the manner in which humans mull over options to finally make a decision [8], it is more natural to use.

When one wants to solve a decision problem, a set of $\mathrm{X}$ possible choices or decisions needs to be ordered, based on the different consequences of each decision. Here, argumentation can be used to define such an ordering. In order to achieve this arguments are to be constructed in favour of, and against each decision. This enables a comparison of possible decisions on the basis of the arguments and their quality or strengths. An argumentation-based decision process can be decomposed into the following steps:

- Constructing arguments in favour of or against each decision in $\boldsymbol{X}$.

- Evaluating the strength of each argument.

- Comparing decisions on the basis of their arguments.

- Defining a pre-ordering on $\boldsymbol{X}$.

Formally, following [8] an argumentation-based decision framework is defined as follows:

An argumentation-based decision framework is a tuple $<\boldsymbol{X}, \boldsymbol{A}, \geq, \quad \Delta_{\text {Princ }}>$ where:

$\boldsymbol{X}$ is a set of all possible decisions.

$\boldsymbol{A}$ is a set of arguments.

$\geq$ is a (partial or complete) pre-ordering on $\boldsymbol{A}$.

$\Delta$ Princ a principle for comparing decisions defines a partial or complete preordering on $\boldsymbol{X}$, defined on the basis of arguments.

Each decision that can be made may have arguments in its favour, and arguments against it. If there are multiple criteria to be considered, an argument in favour of a decision (pro) will be criteria that are positively satisfied. On the other hand, an argument against a decision (con) gives the criteria which are insufficiently satisfied. Arguments may have forces of various strengths [22] that enable an agent to compare different arguments in order to select the best ones, and consequently to select the best decisions. 
Arguments pro/con Let $\boldsymbol{x} \in \boldsymbol{X}[8]$

$-\operatorname{Arg}_{\text {Pro }}(\boldsymbol{x})=$ the set of arguments in $\boldsymbol{A}$ which are in favour of $\boldsymbol{x}$.

$-\operatorname{Arg}_{\mathrm{Con}}(\boldsymbol{x})=$ the set of arguments in $\boldsymbol{A}$ which are against $\boldsymbol{x}$.

In order to support an argument-based comparison of decisions, a principle is required. A simple principle can be defined by merely counting the arguments in favor of each decision. This principle prefers the decision which has more supporting arguments.

An argument supporting a decision takes the form of an explanation. An argument is a 4-tuple $\boldsymbol{A}=\langle\boldsymbol{S}, \boldsymbol{x}, \boldsymbol{g}, \boldsymbol{c}\rangle$ where:

$S$ is the support of the argument,

$\boldsymbol{x}$ is the conclusion of the argument,

$\boldsymbol{c}$ is the criterion which is evaluated for $\boldsymbol{x}$

$\boldsymbol{g}$ represents the manner in which $\boldsymbol{c}$ is satisfied by $\boldsymbol{x}$.

where $\boldsymbol{K}$ represents a knowledge base gathering the available information about the world; $\boldsymbol{X}$ is the set of all possible decision; $\boldsymbol{C}$ is a base containing the different criteria and $\boldsymbol{G}$ is the set of all goals.

To be able to further refine the comparison principle, the strengths of arguments and of the preference relations between arguments are considered. Criteria in $\boldsymbol{C}$ may not have equal importance. The base $C$ is partitioned and stratified into $\boldsymbol{C}=\left(\mathrm{C}_{1} \cup \ldots \cup \mathrm{Cn}\right)$ such that all criteria in $\mathrm{C}_{\mathrm{i}}$ have the same importance level and are more important than criteria in $\mathrm{Cj}$ where $\mathrm{j}<\mathrm{i}$. Each criterion can be translated into a set of consequences, which may not be equally satisfactory. For example, consider the criterion "price" for a product on sale. If the price is 10 or less the user may be fully satisfied, but if it is more than 20 , the user may be unsatisfied. This leads to the fact that there are sets of goals that are either positive $\boldsymbol{G}^{+}$or negative $\boldsymbol{G}^{-}$. A decision to purchase a product is either made or not.

Once it is decided what are considered as pro and con arguments for each possible decision, it is necessary to aggregate them to finally decide what alternative to select. Here, one needs to consider how to manage the potential interactions between arguments that refer to different criteria. Bonnefon and Fargier [23] provide an overview of possible approaches to aggregate sets of pro and cons. These approaches take into account the fact that the arguments are bipolar and qualitative. Amgoud et al. [8] shows that it is possible to make use of classical aggregation operators in their framework. They compare decisions in terms of positive and negative arguments and show that the presented framework can capture different multiple criteria decision rules to select the best decision. The rule for the choice is characterized by the fact that criteria have or do not have the same level of importance.

\section{Framework for Collectivist Decision Support}

To illustrate the framework, a mobile eProcurement application, the Virtual Store, is used as example. It solves the challenges of VSEs such as the replenishment of stock 
using bulk buying. Users are provided with a mobile application to register, login and navigate through a supplier product catalogue. The catalogue contains real-time pricing information and specials that enables VSEs and other retailers to purchase goods in a group. The system allows the formation of collaborative groups where an identified group leader invites group members to be part of the group via the application. Before this can happen, face-to-face agreements are made between group members. A group needs to agree on basic aspects such as the types of products that they will purchase together as well as the threshold amount that they are willing to spend. The goals of the system are to take into account the collectivist nature of the end-user when a decision is make to buy stock in bulk.

The framework is defined next by describing the system architecture, the Virtual Store Agent, and the how collectivist group decision strategies are applied.

\subsection{System Architecture}

The architecture of the system is depicted in Figure 2. At the back-end there is a Virtual Store Server agent and on the mobile device of the users there is a User agent. The Virtual Store Server agent can access the product catalogue of the Virtual Store containing all products that can be purchased.

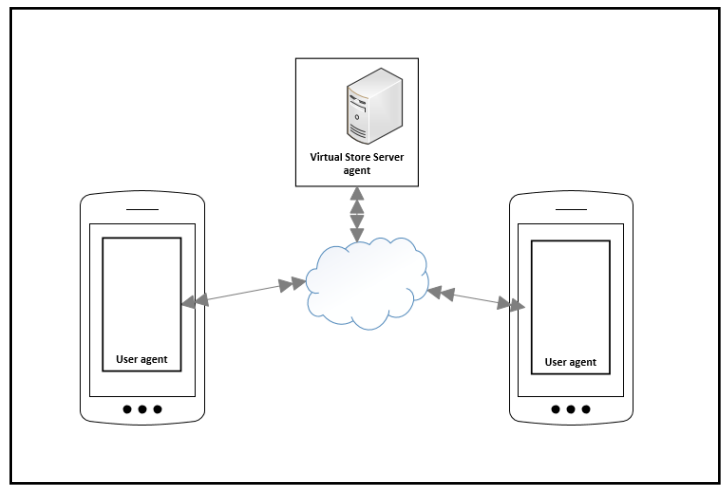

Fig. 2. Virtual store system architecture

These products are sent to the User agent that displays them to users simultaneously, when a session starts. In addition to the image of the products, the Virtual Store agent supports categories that represent particular aspects of products such as price, type or colour. The User agent is defined by subcomponents found in three layers shown in Figure 3 namely the interaction, reasoning and knowledge layers.

In the knowledge layer the User agent stores information about the environment of the user and profile information of other group members and the profile of the user consisting of preferences and goals. The social system component keeps track of the all group members and their relative social position to include this in the group decision computation Knowledge is accumulated over time, as the user interacts with 
the system. The reasoning layer forms a bridge between the knowledge and interaction layers and contains two components namely the argumentation component and decision component. The argumentation component is responsible for the generation of arguments when the human makes choices.

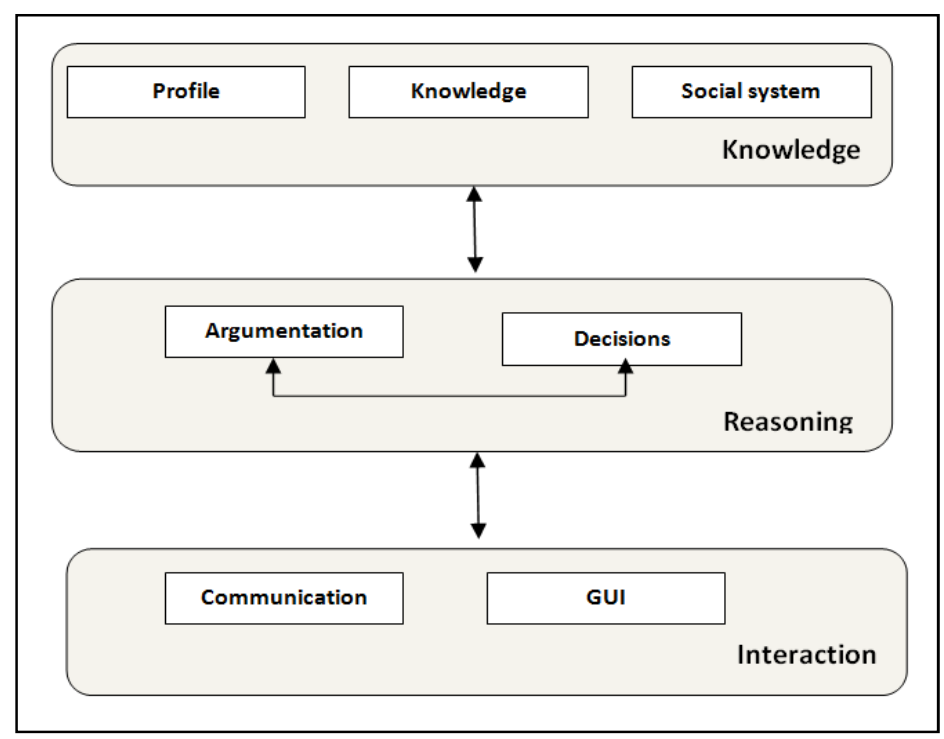

Fig. 3. User agent architecture

The decision component processes the opinions of other User agents to provide a group opinion to the user. The interaction layer is responsible for the communication with other User agents and through the GUI with the user. Using the GUI, users express whether they are in favour of purchasing a product or not. Similarly, they provide their preferences for product categories through this interface.

\subsection{Virtual Store Server Agent}

The Virtual Store Server agent stores product records of a large number of products $\boldsymbol{P}$ that can be purchased. For example, a group of VSE owners collaborate to buy products such as bread and milk in bulk. As a group, they need to create a shopping cart containing products that has been chosen by a group consensus process. They are provided with sets of products. The set of products is defined by $\boldsymbol{P}=\mathrm{p}_{1}, \ldots \ldots \ldots \mathrm{p}_{\mathrm{k}}$, where $\mathrm{k}>2$ is the set of available products.

Each product is described with a finite set of categories or features. The set $\boldsymbol{C}=\mathrm{c}_{1}, \ldots \ldots \ldots \mathrm{c}_{\mathrm{n}}$ contains all the possible categories where $\mathrm{n}>2$. A category may be associated with more than one product. For example, in Table 1, the following three bread products and their four categories are made available by the Virtual Store for bulk buying. 
Table 1. Product categories

\begin{tabular}{|l|l|l|l|}
\hline Price $\left(\mathrm{C}_{1}\right)$ & Quantity $\left(\mathrm{C}_{2}\right)$ & Supplier $\left(\mathrm{C}_{3}\right)$ & Type $\left(\mathrm{C}_{4}\right)$ \\
\hline 100 & 60 & New bakeries & White \\
\hline 100 & 80 & Smiths & Brown \\
\hline 80 & 50 & ABC & White \\
\hline
\end{tabular}

The Virtual Store sends each product and its categories to the User agent, where it is displayed graphically to the user who evaluates it. The application supports strategies for collectivist decision-making, described next.

\subsection{Collectivist Group Decision Making}

The reasoning of the group is now described. A decision protocol is defined to allow the group to reason. To support the collectivist approach to decision-making the decision protocol has two main strategies namely the group customization strategy and group opinion strategy. Then follows a description of the process.

Group Customization Strategy. In-group preferences may be more important than individual preferences in scenarios such as the Virtual Store application. A group customisation strategy allows one member or leader, or various respected members, to customise the Virtual Store application configurations with the in-group's needs, preferences, and goals. Other in-group members can then accept and use the group-level customisation for themselves, thereby saving time and preventing possible uncomfortableness stemming from acting in isolation. It would be possible for group members to set a "personal level" customisation, and they should control which setting will be used in which sitautions. Data can be stored of all in-group preferences, to allow in-group members to stay up to date with group decisions and matters.

Group Opinion Strategy. The group opinion strategy provides the user with opinions of other in-group members while the user is making a decision. This ensures that the user's is not isolated from the group and reduces the time that he may take to consult each member of the in-group regarding their opinions. The group opinion can be presented in a visual manner by giving a graphical representation of categories that may be sized according to the strength of group preference.

The Group Decision Protocol. The group decision protocol is shown in Figure 4. The first step is the formation of a group when a group leader invites members to the group. The invited members accept requests. The group leader and respected members customises the goals and preferences of the group to best represent their requirements. The group leader starts a session to select products for bulk buying by the group. The Virtual Store agent send products to the User agent of each user. Members analyse categories and decide their preferences. Each user expresses his opinion about the product categories through the GUI of the User agent. User agents provide their users a report on the aggregated opinion of the group to determine if an agreement was reached. If so, the decision is made to buy the product. If no agreement is reached, the group opinion formation process starts. Users create new opinions as 
arguments that are exchanged between User agents following an adaptive decisionmaking style. The decision-module of the User agent determines the modified opinion of the group and whether an agreement was reached. This process continues until an agreement is reached to buy or reject the product. If no agreement can be reached, the group leader makes the final decision.

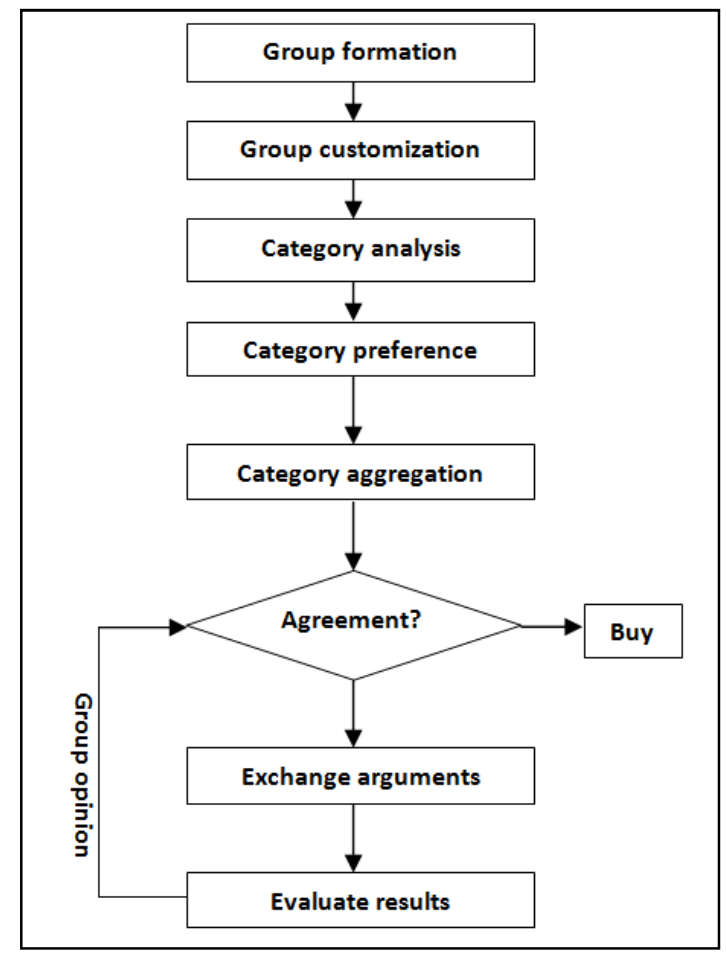

Fig. 4. Group decision protocol

Group decision-making is now described in more detail using the Virtual Store application.

\section{Group Customisation}

Using the group customisation strategy, the group leader of the group of retailers configures the Virtual Store with the products and their related categories the group will prefer to buy in bulk where the set of products for the group is defined by $\mathrm{PG}=\mathrm{p}_{1}, \ldots \ldots \ldots \mathrm{p}_{\mathrm{k}}$, where $\mathrm{k}>2$ is the set of available products and $\mathrm{PG} \in \mathrm{P}$.

\section{Category Analysis}

When making decisions, the user interacts with the system via the GUI, depicted in Figure 2. Each user expresses an opinion about each category of product sent by the Virtual Store Server agent. To ensure ease of use, the opinion is defined by the use of a slider on the GUI of the application. 
Users provide their preferences and rejections for each category. This is done through a value, in the opinion range $\mathrm{Op}=[0,100]$ for each category associated with the product. In this context, the best preferred option is assigned a score of 100, and the least preferred a score of 0 . This range can be customised according to the specific scenario. Assigning a value more than 50 to a product means that the product should be bought in bulk by the group.

\section{Category Preference}

The preferences of a user is defined as follows:

$\mathrm{U}_{\mathrm{i}}=\left\{\mathrm{s}_{\mathrm{ij}}\right\}$ is the score assigned by user $\mathrm{i}$ to category $\mathrm{j}$. This preference represent the user's opinion of a product. For example $U_{1}=\{70,20,90,70\}$ gives the opinions of user 1 for each category $\mathrm{c}_{1}$ to $\mathrm{c}_{4}$ of a single product. The user gives a high preference to the baker (c3) Shown in Table 1 that provides the bread. The user may be able to change his opinion, based on what the group decides.

The overall score for each option, $\mathrm{Si}$ is given by summing the preference score for option i on criterion $\mathrm{j}$.

$\mathrm{Si}=\sum_{j=1}^{n} s i j / n$

For user $1, S_{1}=63$, indicating the user is in favour of buying the product.

After being provided a report on the ratings of other group members, a user can revise his opinion.

\section{Category Aggregation}

It is very important for each user to know the opinion of the group as the user may want to revise his opinion to accommodate the preferences of the group.

For example, given are the opinions of a set of 4 users for a product and their total scores:

$$
\begin{aligned}
& \mathrm{U}_{1}=\{70,20,90,70\} \quad \mathrm{S}_{1}=63 \\
& \mathrm{U}_{2}=\{50,30,20,65\} \quad \mathrm{S}_{2}=42 \\
& \mathrm{U}_{3}=\{70,40,20,80\} \quad \mathrm{S}_{3}=53 \\
& \mathrm{U}_{4}=\{50,30,30,70\} \quad \mathrm{S}_{4}=45
\end{aligned}
$$

The opinion of the group $\mathrm{Op}_{\mathrm{i}}$ is calculated for each category as follows:

$$
\begin{aligned}
& \mathrm{op}_{\mathrm{i}}=\sum_{j=1}^{n} \text { oij } / n \\
& \mathrm{op}_{\mathrm{i}}=\{60,30,40,72\} \quad \mathrm{S}_{\mathrm{i}}=50
\end{aligned}
$$

The opinion of user 1(63) is thus out of bounds with the group $(42,53,45)$.

\section{Group Opinion}

As collectivist consumers prefer an adaptive decision-making style, they should be supported to change their mind when confronted with the opinion of the group. The focus of this research thus to support a group to reach consensus on a joint decision. Each member of the group can give their opinion, and change their mind when needed. This gives these consumers more confidence when making decisions, as they feel that they are complying to the preferences and goals of the group.

For this purpose, arguments pro and con to be used with multi-criteria decision analysis are now defined. 
pro $\mathrm{p}_{\mathrm{g}}$ is a pair $\left((\mathrm{c}, \mathrm{o}), \mathrm{p}_{\mathrm{g}}\right)$ where $\mathrm{c} \in \boldsymbol{C}$ and $\mathrm{o} \in \mathrm{O}$ and $\mathrm{o}>50$.

con $\mathrm{p}_{\mathrm{g}}$ is a pair $\left((\mathrm{c}, \mathrm{o}), \mathrm{p}_{\mathrm{g}}\right)$ where $\mathrm{c} \in \boldsymbol{C}$ and $\mathrm{o} \in \mathrm{O}$ and $\mathrm{o} \leq 50$.

The pair (c,o) represents the opinion of a user for a given category of a product. If the opinion is more than 50 , the argument is in favour to buy the product, and if the opinion is less or equal to 50 , the opinion is in favour to reject the product.

When a user changes his opinion about a given category, the initial argument is removed and replaced by another. For example, if the user decides to give a low preference to the baker, he can decrease the opinion to 30 from 90 . The argument $\left(\left(c_{3}\right.\right.$, $90), p)$ is replaced by $\left(\left(c_{3}, 30\right), p\right)$. The first argument is thus completely removed from the set of arguments of the user.

User 1 views the opinion of the group provided by his User agent and sees that the group is not in favour of the product. Similarly, user 3 sees that he is also out of the norms of the group. Both users modify their opinion by providing new arguments as follows: $U_{1}=\left(\left(c_{3}, 30\right), p\right)$ and $U_{3}=\left(\left(c_{2}, 30\right), p\right)$ resulting in the following set of opinions:

$$
\begin{aligned}
& \mathrm{U}_{1}=\{70,20, \mathbf{3 0}, 70\} \quad \mathrm{S}_{1}=48 \\
& \mathrm{U}_{2}=\{50,30,20,65\} \quad \mathrm{S}_{2}=42 \\
& \mathrm{U}_{3}=\{70,30,20,80\} \quad \mathrm{S}_{3}=50 \\
& \mathrm{U}_{4}=\{50,30,30,70\} \quad \mathrm{S}_{4}=45
\end{aligned}
$$

The opinion of the group changes to:

$$
\mathrm{op}_{\mathrm{i}}=\{60,28,25,72\} \quad \mathrm{S}_{\mathrm{i}}=46
$$

The group is now in agreement to reject this product.

\section{Conclusion}

This paper proposes a framework for the Virtual Store application to enable a group of users such as VSE owners to create a shared shopping cart containing products that have been agreed to as a group. In the framework, users are provided with products and related criteria over which to define their preferences, which are the strengths of their arguments that they present. A joint group opinion is formed which is reported to all users to assist them to feel part of the group and to ensure that they can comply to the group opinion if needed. The architecture consists of two parts namely the Virtual Store server agent and the User agent through which users interact with the system.

To the knowledge of the authors, the framework to address collectivist decisionmaking is the first of its kind. The framework is a step towards supporting collectivist beliefs and behaviours relating to decisions and group opinions by using argumentation-based decision for a multi- criteria decision problem. This approach naturally support the manner in which opinions can be changed as new facts come to light.

Next research is addressing the refinement of features to be addressed in the decision framework, the implementation of a prototype and the evaluation of the framework. 
Acknowledgments. The support of the National Research Foundation (NRF) under grant number 81201 toward this research is hereby acknowledged. Opinions expressed and conclusions arrived at are those of the authors and not necessarily to be attributed to the NRF.

\section{References}

1. Economic Report on Africa, "Governing development in Africa - the role of the state in economic transactions", http: / / www . uneca.org/era2011/ (accessed February 8, 2012)

2. LeFebvre, R., Franke, V.: Culture Matters: Individualism vs. Collectivism in Conflict Decision-Making. Societies 3(1), 128-146 (2013)

3. e-spaza (2011), http: / / www . e-spaza. com/ ?p=251 (accessed February 9, 2012)

4. Radebe, K.: R7bn worth of untapped township potential (December 27, 2012), http: / / www . moneyweb. co.za/moneyweb-mybusiness / r7bn-worth-ofuntapped-township-potential (date accessed: June 19, 2013)

5. Dörflinger, J., Friedland, C., Mengistu, M., Merz, C., Stadtrecher, S., Pabst, K., de Louw, R.: Mobile commerce in rural South Africa - Proof of concept of mobile solutions for the next billion mobile consumers. In: Proceedings of the 10th IEEE International Symposium on a World of Wireless, Mobile and Multimedia Networks - WOWMOM 2009, Kos Island, Greece, June 15-19 (2009)

6. Ntawanga, F., Eloff, J.H.P., Ngassam, E.K., Kandie, W.: User Experience Evaluation of a Lightweight Mobile EProcurement Application for Rural Small-Scale Retailers. In: Proceedings of the Fourth International IEEE EAI Conference on eInfrastructure and eServices for Developing Countries, Yaoundé, Cameroon, November 12-14 (2012)

7. Dhar, M.: Brand Management 101-101 Lessons from Real World Marketing. John Wiley \& Sons (Asia) Pte Ltd., Singapore (2007)

8. Amgoud, L., Bonnefon, J.-F., Prade, H.: An argumentation-based approach to multiple criteria decision. In: Godo, L. (ed.) ECSQARU 2005. LNCS (LNAI), vol. 3571, pp. 269-280. Springer, Heidelberg (2005)

9. Hofstede, G., Hofstede, G.J., Minkov, M.: Cultures and organizations. McGraw-Hill, London (1991)

10. Martin, R., Hewstone, M.: Social-influence processes of control and change: Conformity, obedience to authority and innovation. In: Hogg, M.A., Cooper, J. (eds.) The SAGE Handbook of Social Psychology, pp. 347-366. Sage, London (2003)

11. Hofstede, G.: Culture's consequences: International differences in work-related values. Sage Publications, Incorporated (1980)

12. Usunier, J.C.: International and Cross-Cultural Management Research. Sage, London

13. Yetim, F.: Cultural Aspects of Group Support Systems. In: Zimmermann, H., Schramm, V. (eds.) Knowledge Management and Communication Systems, Proceedings of 6th International Symposium on Information Science, ISI 1998, Prague, November 4-7, Universitätsverlag, Konstanz (1998)

14. Khaled, R., Biddle, R., Noble, J., Barr, P., Fischer, R.: Persuasive interaction for collectivist cultures. In: Piekarski, W. (ed.) The Seventh Australasian User Interface Conference (AUIC 2006), pp. 73-80 (2006)

15. Güss, D.: Decision making in individualistic and collectivist cultures. In: Lonner, W.J., Dinnel, D.L., Hayes, S.A., Sattler, D.N. (eds.) OnLine Readings in Psychology and Culture, Western Washington University, Department of Psychology, Center for CrossCultural Research (2002) 
16. Yeboah, A.: The Influence of Culture on Consumer Choice: A Case of the Fast Moving Consumer Goods In Ghana. International Journal of Business and Management Tomorrow 2(2) (February 2012)

17. Salo, A., Hämäläinen, R.P.: Multicriteria Decision Analysis in Group Decision Processes. In: Kilgour, D.M., Eden, C. (eds.) Handbook of Group Decision and Negotiation. Springer, Dordrecht (2010)

18. Kilgour, D., Chen, Y., Hipel, K.: Chapter 11 - Trends in Multiple Criteria Decision Analysis. In: Greco, S., Ehrgott, M., Figueira, J.R. (eds.). XVI. International Series in Operations Research \& Management Science, vol. 142, p. 412 (2010)

19. Ouerdane, W., Maudet, N., Tsoukias, A.: Chapter 7 - Argumentation Theory and Decision Aiding. In: Greco, S., Ehrgott, M., Figueira, J.R. (eds.) XVI. International Series in Operations Research \& Management Science, vol. 142, p. 412 (2010)

20. Ouerdane, W., Maudet, N., Tsoukiàs, A.: Argumentation theory and decision aiding. In: Figueira, J., Greco, S., Ehrgott, M. (eds.) International Series in Operations Research and Management Science, 1,Trends in Multiple Criteria Decision Analysis, vol. 142, pp. 177-208 (2010)

21. Chesnevar, C., Maguitman, A.G., Loui, R.P.: Logical Models of Argument. ACM Computing Surveys 32(4), 337-383 (2000)

22. Ouerdane, W., Dimopoulos, Y., Liapis, K., Moraitis, P.: Towards automating decision aiding through argumentation. Journal of Multi-Criteria Decision Analysis 18, 289-309 (2011)

23. Bonnefon, J.-F., Fargier, H.: Comparing sets of positive and negative arguments: Empirical assessment of seven qualitative rules. In: Proc. of 17th European Conf. on Artificial Intelligence. IOS Press (2006)

24. Triandis, H.C.: Individualism-collectivism and personality. Journal of Personality 69, 907-924 (2001)

25. Bricks, D.A.: Bunders in International Business, 4th edn. Blackwell (2006)

26. Tapia Garcia, J.M., del Moral, M.J., Martínez, M.A., Herrera-Viedma, E.: A consensus model for group decision making problems with linguistic interval fuzzy preference relations. Expert Syst. Appl. 39(11), 10022-10030 (2012)

27. Isherwood, D., Coetzee, M.: Towards Trust and Reputation for E-Commerce in Collectivist Rural Africa. In: International Symposium on Human Aspects of Information Security \& Assurance (HAISA 2012), Crete, Greece, June 6-8 (2012) 